\section{La Révolution française}

Cahiers de l'Institut d'histoire de la Révolution française

12 | 2017

Pratiques et enjeux scientifiques, intellectuels et politiques de la traduction (vers 1660-vers 1840)

\title{
Helen-Maria Williams, médiatrice culturelle dans La Décade philosophique
}

\section{Bernard Gainot}

\section{CpenEdition}

Journals

Édition électronique

URL : http://journals.openedition.org/lrf/1754

DOI : $10.4000 /$ Irf. 1754

ISSN : 2105-2557

Éditeur

IHMC - Institut d'histoire moderne et contemporaine (UMR 8066)

Référence électronique

Bernard Gainot, « Helen-Maria Williams, médiatrice culturelle dans La Décade philosophique », La

Révolution française [En ligne], 12 | 2017, mis en ligne le 15 septembre 2017, consulté le 19 avril 2019

URL : http://journals.openedition.org//rf/1754 ; DOI : 10.4000/Irf.1754

Ce document a été généré automatiquement le 19 avril 2019

(c) La Révolution française 


\title{
Helen-Maria Williams, médiatrice culturelle dans La Décade philosophique
}

\author{
Bernard Gainot
}

1 Les récits de prises de contact des Européens avec les populations autochtones présentent l'acte de traduire comme une pratique de médiation culturelle mobilisant certes les instruments usuels comme les dictionnaires, mais également des capacités autres que l'écriture: langage corporel par le geste, recours à des supports matériels. À titre d'exemple, voici comment Étienne de Flacourt, au xVII ${ }^{\mathrm{e}}$ siècle, pose la traduction comme centrale dans le contact avec les populations de Madagascar :

Nous nous appliquâmes à l'intelligence de la langue du pays, en quoi nous avons eu beaucoup de peine, car le dictionnaire qu'on nous prêta dans le navire, outre qu'il comprenait fort peu de mots, n'était ni en bon ordre, ni assuré ; et il y a grande différence de la prononciation à l'écriture; et c'est autre chose de savoir la signification d'un mot séparé et connaître la force dans la construction, et le distinguer, et entendre dans le discours des naturels du pays. Voilà pourquoi il nous fallut avec beaucoup de peine assembler les interprètes qui se trouvaient bien empêchés à trouver des mots pour expliquer notre foi en un pays où on ne parle point de religion ${ }^{1}$.

2 La pratique de la traduction a une dimension anthropologique : c'est toute la question de la transposition, qui suppose un jeu mouvant avec l'altérité. Enfin, elle entretient un rapport ambigu avec l'information, dans le double sens de la collecte du renseignement, et de la diffusion de connaissances dans l'espace public. L'importance prise par les truchements dans l'entreprise de colonisation montre toutes ces dimensions, une pratique et des savoirs liés à un statut spécifique, portés par une personne ou un groupe de personnes. Le truchement est l'intermédiaire, attaché au service du traducteur attentif à la collecte des signes indispensables à cette double démarche d'observation et de transposition.

3 Ce bref retour sur les Relations ${ }^{2}$ des premiers voyageurs, et sur l'acculturation spécifique à l'entreprise de colonisation, peut sembler très éloigné du propos ultérieurement 
développé. Il a toutefois l'avantage de mettre l'accent sur l'inscription d'une pratique aux implications multiples (la traduction) dans une représentation mouvante des groupes humains (la ou les civilisations). Ainsi donc, l'interrogation sur la transposition des langages est-elle au cœur de la préhistoire de l'anthropologie. Dans une tout autre perspective que l'eschatologie qui portait les missionnaires et les explorateurs, un courant de pensée comme l'idéologie, dans la décennie de la Révolution française, accorde une grande attention au codage des signes écrits, en une époque où la notion de civilisation est une clef interprétative de la science de l'Homme en formation ${ }^{3}$.

Par là même, la question de la traduction est essentielle dans le journal des Idéologues, $L a$ Décade philosophique, littéraire et politique 4 . Comme il n'était pas question de présenter un tableau de l'ensemble de l'entreprise de traduction de ce journal, qui occupe une place considérable, j'ai mis l'accent sur une personnalité de premier plan au sein de ce braintrust de la République directoriale, qui tient le rôle actif de traductrice, et le rôle passif d'auteur traduit. Elle illustre bien la position de médiatrice, par son origine anglaise en résidence française, alors que les deux nations sont engagées dans une guerre sans merci aux implications mondiales. Elle permet enfin d'interroger la lecture très fréquemment admise - c'est même devenu un lieu commun - de l'idéologie officielle de «la Grande Nation » comme expression d'une idéologie nationaliste, et non nationale. Dans l'écart, à mon sens déterminant, entre les deux qualificatifs, se tiennent véritablement tous les enjeux d'une grammaire des civilisations, qui est à l'horizon des entreprises de traduction de l'époque.

\section{Qui est Helen-Maria Williams ?}

5 Helen-Maria Williams est née à Londres le 17 juin $1761^{5}$. Elle fut très tôt orpheline de père : sa mère et sa sœur animent un salon politico-littéraire à Londres, que fréquentent des personnalités «radicales» (ou dissenters) : Richard Price, Joseph Priestley, Josiah Wedgwood, Granville Sharp, Mary Wollstonecraft ou Anna Laetitia Barbauld. Ce milieu, qui milite pour la réforme électorale, la tolérance religieuse et l'abolition de la traite des noirs, manifeste un très grand intérêt pour les changements intervenus en France après 1789 et se retrouve bientôt à la "Revolution Society ». En butte aux attaques des contrerévolutionnaires, menés par Edmund Burke, Mme Williams et ses filles s'installent définitivement sur le continent à partir de l'été 1791. C'est à Paris que se reconstitue un milieu « radical » anglo-saxon, à l'hôtel de White, chez John-Hurford Stone, qui a fondé une éphémère Société des Amis des droits de l'Homme, entre le 19 novembre 1792 et octobre 1793 ; on y trouve une cinquantaine de personnes, dont Joël Barlow, Thomas Paine, Mary Wollstonecraft et, bien entendu, Helen-Maria Williams, qui est liée à Stone depuis son premier séjour à Paris, en 1790.

6 Ces exilés sont en relations étroites avec le groupe girondin: Stone est très proche de Kersaint et de Servan, Mlle Williams fréquente le salon de Manon Roland, et le couple est en relations très intimes avec Français de Nantes, Lanthenas et Garran-Coulon. Ce qui les rend bien vite suspects à partir de l'été 1793 : le 11 octobre 1793, les dames Williams sont arrêtées lors de la grande rafle des Anglais de Paris. Elles sont libérées un mois plus tard, mais doivent de nouveau quitter Paris pour se conformer au décret du 27 germinal an II/16 avril 1794, qui éloigne de la capitale les résidents étrangers ${ }^{6}$. La famille Williams connaît une période difficile, marquée par un nouvel exil, en Suisse cette fois-ci ${ }^{7}$. Le couple Stone-Williams rétablit sa situation dès les lendemains de Thermidor, mais surtout 
il s'insère durablement dans les cercles dirigeants de la République directoriale, par ses relations qui superposent plusieurs époques, comme nous le verrons ultérieurement, grâce à une pratique renouvelée de la sociabilité littéraire, politique et philosophique (pour pasticher le titre du journal qui va être le relais d'influence de cette sociabilité). Le salon d'Helen-Maria, au 433 de la rue de Verneuil, est l'un des trois salons parisiens (avec celui de Germaine de Staël et celui de Sophie de Condorcet à Auteuil) qui brassent les élites républicaines. On y trouve Bancal des Issarts, Lanthenas, Siéyès, Servan, Griffet de Labaume, Français de Nantes, Thomas Paine et, surtout, trois personnalités de premier plan, l'abbé Grégoire, Jean-Baptiste Say, et Paul-Henri Marron, pasteur de l'ambassade de Hollande $^{8}$. Fréquente également le salon d'Helen-Maria Williams, Jean-Baptiste Salaville, qui a participé à cette entreprise. Quant à Say et à Grégoire, ce sont deux très proches amis d'Helen-Maria (Grégoire se considère même comme le père de substitution de la jeune protestante). Par leur intermédiaire, des relations sont entretenues dans les centres intellectuels du pouvoir : la classe des Sciences morales et politiques de l'Institut national, la Décade dont Say est l'un des rédacteurs, et la Société des Amis des noirs et des colonies, qui apparait comme un prolongement " philanthropique » de ce milieu. Nous allons nous efforcer d'en établir les synergies et les cohérences, par la médiation des traductions d'ouvrages.

Le contexte dans lequel s'insèrent les transferts linguistiques dans le journal La Décade est fortement marqué par la guerre avec l'Angleterre. L'attitude envers l'Angleterre des élites républicaines françaises est empreinte d'une forme de dépit amoureux ${ }^{9}$. On cite abondamment les ténors de l'opposition, on traite des affaires britanniques presque comme s'il s'agissait d'affaires intérieures (relations des débats aux Communes et suivi de l'actualité éditoriale). Vraisemblablement, la revue disposait d'informateurs dans le pays. Les spécialistes de l'Angleterre ne sont pas des collaborateurs de second rang; JeanBaptiste Say, qui a séjourné à plusieurs reprises en Angleterre à la veille de la Révolution, qui connaît bien la langue et le pays, ainsi que Antoine-Prosper Lottin dit «Lottin le jeune» (1739-1812), qui est un libraire. Il signait ses articles des initiales E. B., ce qui voulait dire Encyclopédie britannique ${ }^{10}$.

8 Enfin, Joseph-Thérèse Masclet a écrit l'article essentiel, « Oligarchie anglaise » (10 brumaire an VIII/1 $1^{\mathrm{er}}$ novembre 1799) qui rend bien compte de l'ambivalence des libéraux français à l'égard de la sœur siamoise qui a mal tourné. Masclet, qui a vécu en Angleterre entre 1793 et 1796, est un proche de Charles-Bernard Wadström, autre médiateur essentiel, mais il renvoie surtout à l'entourage de Mirabeau, et au journal Analyse des papiers anglais, importante entreprise de traduction prérévolutionnaire, et préfiguration du projet des rédacteurs de La Décade. Jean-Baptiste Say fut le secrétaire de Mirabeau à cette époque, et l'un de ses interprètes. Les interlocuteurs privilégiés de La Décade sont donc tout naturellement les transfuges du radicalisme anglais installés sur le territoire de la République. L'intérêt porté à la Grande-Bretagne n'empêchait nullement par ailleurs les sympathies exprimées envers les Irlandais Unis (Theobald Wolfe Tone ou Nicolas Madgett, qui sont des informateurs et des interprètes auprès du Directoire) tandis que Henry Grattan ou James Napper-Tandy sont adulés comme des héros ${ }^{11}$.

$9 \quad$ La Décade offre en outre à ses lecteurs des traductions d'ouvrages en d'autres langues européennes: le journal présente le 30 germinal an IX/20 avril 1801 la Bibliothèque germanique, un ouvrage qui est une bibliothèque "choisie et complète " destinée à présenter « aux personnes qui ne savent pas la langue allemande la réalité des richesses immenses de la littérature allemande ». Parmi les initiateurs de cette expérience, on 
trouve Griffet-Labaume et Charles Cramer ${ }^{12}$. L'« art de la traduction » est une dimension centrale de l'entreprise de presse, et le journal se pose en modèle : ainsi fait-il en prairial an VII/juin 1799 la promotion de Phraseologica anglo-germanica, une grammaire anglaiseallemande qui est un recueil de plus de 50000 phrases tirées des meilleurs auteurs classiques anglais, mises par ordre alphabétique et fidèlement traduites en allemand. À l'inverse d'un modèle hermétique et clos sur la sphère nationale, la «Grande Nation » se pose ainsi en médiatrice de la communication européenne. On connaît encore le rôle que joue Ginguené dans la diffusion de la littérature italienne; c'est lui qui fait le compterendu de la publication des Euvres complètes de Machiavel par Toussaint Guiraudet, qui allait renouveler entièrement la lecture républicaine du penseur florentin ${ }^{13}$.

\section{Helen-Maria Williams, médiatrice} traductrice (en anglais) et écrivain traduite en français, vecteur d'une nouvelle pratique narrative. Elle a notamment traduit Paul et Virginie ${ }^{14}$ en anglais, mais sa grande réalisation reste la traduction, à l'époque de la Restauration, des Voyages d'Alexandre de Humboldt en Amérique ${ }^{15}$. Parallèlement, les traductions de ses ouvrages font l'objet de comptes rendus approfondis et élogieux: le Nouveau voyage en Suisse qui a été traduit par JeanBaptiste Say ${ }^{16}$, et Aperçu de l'état, des moeurs et des opinions dans la République française, vers la fin $d u X^{X V I I I}$ siècle, traduit par Sophie Grandchamp ${ }^{17}$, que connaît Helen-Maria depuis l'époque où toutes deux fréquentaient le salon de Manon Roland. C'est Jean-Baptiste Say qui en fait un long commentaire, en deux rubriques. La première partie présente l'échec de la Révolution de Naples ; l'auteure prétend avoir recueilli ses informations auprès d'un réfugié napolitain arrivé à Marseille ${ }^{18}$. Ce témoignage relève de l'histoire immédiate : c'est l'une des premières analyses "à chaud » de l'expérience républicaine, avant même le Saggio storico sulla rivoluzione di Napoli de Vincenzo Cuoco, publié en $1801^{19}$. L'interprétation de la révolution de Naples est originale :

Elle a été embrassée et dirigée par tout ce qu'il y avait dans tous les ordres du royaume, de gens recommandables par des talents éminents ou des vertus reconnues, contre la plus vile canaille de l'Europe, sans exception, et contre un Roicanaille, allié naturel de cette populace. Les mœurs, les goûts révolutionnaires étaient dans ceux contre qui la révolution s'est faite, tandis que l'amour de la tranquillité, le soin des propriétés étaient dans cette portion de la noblesse, du clergé, des jurisconsultes et des gens de lettres qui l'ont embrassée; de manière qu'elle a eu véritablement l'air d'une cessation de mesures révolutionnaires, mesures que la contre-révolution a renouvelées avec fureur.

11 C'est une lecture qui rend l'événement transposable dans la grille de lecture des républicains post-thermidoriens. Il y a une traduction du fait pour le rendre assimilable dans une autre conjoncture politique. L'enjeu de cette traduction était la transposition de la Terreur du camp républicain au camp royaliste, avec l'avertissement sous-jacent qui était déjà celui de Benjamin Constant en $1797^{20}$ : toute nouvelle convulsion populaire entraînerait une réaction sanglante; des barques viennent chercher les patriotes napolitains pour les conduire au tribunal spécial et à la mort :

[...] le bruit des rames, lorsque ces barques approchaient, portait la terreur dans leurs âmes; de même que sous le règne de Robespierre, les infortunés qui remplissaient les prisons, entendaient avec la stupeur de l'effroi, l'appel qui se faisait chaque soir et à la suite duquel ils étaient conduits à la Conciergerie et à l'échafaud. 


\section{de la République napolitaine :}

Si nous en croyons même l'historien que nous analysons, ces causes ne sont pas les seules qui amenèrent une sanglante catastrophe, et l'on doit regarder comme la plus décisive la volonté du Directoire français, qui ne voulait pas que la république napolitaine existât. Il faut supposer que l'auteur a de bonnes raisons pour fonder cette opinion ; mais plusieurs lignes de points dans cet endroit, indiquent au lecteur qu'il a jugé à propos de les taire ${ }^{21}$.

Sous prétexte d'une réplique au pamphlet de D'Ivernois ${ }^{22}$, qui vantait la stabilité du gouvernement anglais, il s'agit certes de justifier le coup d'état de brumaire, mais bien plus encore la supériorité du système républicain sur une nation atteinte de l'hybris de la puissance :

Nous connaissons trop bien l'excès de l'orgueil et le manque de sagesse du gouvernement anglais, pour croire qu'il se détermine à prendre des mesures aussi salutaires. Nation guerrière et ambitieuse, puissance commerciale, la passion des conquêtes, le besoin de dominer, la soif d'un gain monopole, se réuniront pour éloigner de sa vue ses véritables intérêts, et la porter sans cesse à ces expéditions qui dépouillent les nations, dépeuplent la terre et ne peuvent manquer de lui devenir aussi funestes à la fin, que les croisades le furent à la plupart des États de l'Europe ${ }^{23}$.

L'histoire immédiate et la lecture politique ne sont pas les seules originalités de l'essai de Mlle Williams. Jean-Baptiste Say souligne la nouveauté du procédé narratif :

$\mathrm{M}^{\text {elle }}$ Williams a su ajouter un nouveau prix à l'intérêt de cette histoire, par des détails pleins de sentiment et de vérité, d'où l'on peut conjecturer que si elle ne s'était pas vouée à la poésie et à la politique, elle aurait accru la réputation que les femmes ses compatriotes se sont acquises dans la narration des romans. Nous l'invitons à s'exercer dans ce genre où brille avec tant d'avantages la délicatesse des sentiments, si naturelle à son sexe. Ses écrits ont de quoi plaire aux hommes qui pensent fortement; mais elle n'oubliera pas sans doute que les femmes ont aussi quelques droits sur les productions d'une plume qui peut suivre les mouvements les plus fugitifs du cœur, aussi facilement qu'elle sait tracer le tableau des grands bouleversements politiques et la peinture des mœurs d'une nation.

Il déplace ainsi la critique sur les enjeux de la traduction, le point de vue d'une anglaise sur les événements de France :

On pourrait croire qu'un tableau où sont représentés des objets que nous avons sous nos yeux, est plus propre à piquer la curiosité des étrangers que la nôtre ; cependant, nous ne sommes pas toujours placés au vrai point de vue pour bien nous juger, et il peut être piquant de connaître ce que les étrangers pensent et disent de nous.

Il est donc fondamental de mettre en avant l'écriture, l'effet de langue, comme témoignage des mœurs. La contribution de Helen-Maria Williams à la promotion d'une nouvelle sensibilité littéraire se remarque surtout à travers ses propres comptes rendus de romans anglais. Elle ne tarit pas d'éloges sur Eugenio et Virginia, roman de Louise Brayer de Saint-Léon ${ }^{24}$. Il est susceptible d'instruire autant que de plaire. Certes, on fait mine de cultiver un nationalisme littéraire, et de se lamenter de la vogue des romans anglais et allemands, mais c'est une position convenue, et qui n'engage pas les auteurs dans la voie d'une déconsidération du genre.

17 Toujours dans le même extrait, il y a des considérations sur les règles de la vraisemblance. C'est ce qui fait que, selon Helen-Maria Williams, Richardson est au-dessus de Rousseau. Helen-Maria Williams rejoint absolument un petit opuscule fort peu connu, 
mais tout à fait intéressant du marquis de Sade, Idée sur les romans, paru en 1799. Pour Sade, la Nouvelle Héloïse est un « livre sublime» :

Puisse cette vérité faire tomber la plume des mains de cette foule d'écrivains éphémères qui, depuis trente ans, ne cessent de nous donner de mauvaises copies de cet éternel original. Qu'ils sentent donc que, pour l'atteindre, il faut une âme de feu comme celle de Rousseau, un esprit philosophe comme le sien - deux choses que la Nature ne réunit pas dans le même siècle ${ }^{25}$.

Helen-Maria Williams contribue également à la diffusion des idées de ceux qui fréquentent son salon, au premier rang desquels le philanthrope d'origine suédoise CarlBernhard Wadstrom, qui a passé de longues années en Angleterre, où il a publié son Essay on colonization ${ }^{26}$. Républicain dans le même sens qu'Helen-Maria Williams, Jean-Baptiste Say ou Charles Theremin, il doit également fuir le régime d'exception anglais et se réfugier en France. Cheville ouvrière de la seconde Société des Amis des Noirs, qui diffuse ses théories sur la " colonisation libre ", ou " colonisation nouvelle ${ }^{27}$ ", il meurt à Paris le 15 germinal an VII/4 avril 1799. C'est Helen-Maria qui rédige sa notice nécrologique ${ }^{28}$ :

Wadstrom publia à Londres un gros volume in/4, renfermant le résultat de ses observations sur l'Afrique, et un grand nombre de notes, de renseignements utiles sur la colonisation en général, et sur celle de la côte d'Afrique en particulier. Des deux ou trois exemplaires de cet ouvrage qu'il avait apportés d'Angleterre à Paris, il ne lui en restait qu'un seul; et la difficulté des communications ne lui permettait pas d'en faire venir d'autres. Bonaparte, au moment de partir pour l'Égypte, désira d'emporter cet ouvrage, et fit demander à Wadstrom de lui indiquer un moyen de se le procurer ; Wadstrom n'en connut point d'autre que de faire passer au général un hommage gratuit du dernier exemplaire qu'il possédait.

C'est un personnage aujourd'hui bien oublié, mais qui joua à l'époque un rôle considérable pour redéfinir une politique française de présence outre-mer, alors que, l'esclavage ayant été aboli, il faut trouver des alternatives à la traite des hommes et au commerce avec les puissants empires africains. C'est la colonisation nouvelle, dont Wadstrom a décrit les ébauches, aussi bien pour les établissements côtiers fondés sur le commerce libre, sur le modèle de la Sierra Leone, que pour les grands voyages en direction des terres inconnues de l'Afrique intérieure, à travers lesquels on cherchait à faire la jonction avec la Haute-Égypte. En outre, Helen-Maria Williams pouvait se sentir en profonde communauté d'esprit avec ce républicain suédois passé par l'Angleterre, condamné pour trahison par le gouvernement de ce pays, qui trouva en France une terre de refuge. Dans l'expérience unique de l'exil, la traduction est aussi un trait d'union entre les opprimés de tous les pays, un rappel du patriotisme de conviction, et de l'écart avec le nationalisme qui semble alors submerger l'opinion française. Mais la traduction n'est pas qu'une entreprise politique, c'est aussi une expérience unique de la langue.

\section{L'art de la traduction}

La civilisation, pour les Idéologues, est un processus universel qui transcende les particularismes nationaux et les différences ethniques. Mais, dans l'avancement de ce processus, l'étude des langues ne semble pas faire dans l'apprentissage des progrès aussi rapides que pour les sciences et les arts. «On commence à sentir que la perfection du langage est aussi nécessaire pour les opérations de l'esprit, que la perfection des instruments pour les travaux mécaniques ${ }^{29}$. Les difficultés dans ce domaine ne tiennent pas seulement à l'insuffisante qualité des instruments ou de l'éducation, mais aussi à 
l'incomplétude de l'armature conceptuelle: la nomenclature grammaticale n'est encore fixée dans aucun pays.

Il est bien à désirer que cette incertitude cesse, et que la science des mots, qui a tant d'influence sur la science des choses, et à laquelle tient de si près la justesse des idées, ait un type invariable. On l'a trouvé pour les mesures de toute espèce. Dans une science moins positive, la chimie, on a rencontré des mots-définitions dont un assentiment presque général a prouvé le mérite et l'exactitude. Après les travaux de Dumarsais, de Condillac, et de Sicard, on s'étonne que l'on ne soit pas parvenu encore au même résultat pour la grammaire. On entrevoit cependant qu'il serait possible de généraliser, de simplifier les principes, de manière à les rendre applicables à tous les idiomes.

Toutes les langues diverses étant les interprètes des mêmes opérations de l'esprit, elles paraissent sous ce point de vue susceptibles d'être toutes ramenées à des principes généraux et communs. Ainsi considérées, ce que l'on dit de l'une s'appliquera également à l'autre, de manière qu'un travail vraiment philosophique fait sur une seule, serait, à très peu de chose près, un type pour toutes. Alors, l'arbitraire disparaîtrait nécessairement d'une nomenclature toujours fondée en raison. Alors on pourrait espérer qu'elle serait invariable; on s'entendrait du moins; ce qui n'arrive pas aujourd'hui, en passant dans sa propre langue d'une grammaire à une autre, et bien moins encore quand on essaie de comparer entre elles les grammaires des divers pays ${ }^{30}$.

21 Autour de la pratique de la traduction, il se construit, dans ces milieux qui se veulent porteurs des valeurs républicaines de la régénération des mœurs par le sentiment et par le langage, une véritable "grammaire des civilisations ». Instrument de médiation entre des cultures dont il s'agit de dépasser les antagonismes, pour en percevoir les signes universels, produit d'une réflexion sur la langue («mal nommer les choses, c'est contribuer au malheur du monde" dira Camus, en digne héritier du projet des Idéologues), c'est également la pratique narrative d'une nouvelle sensibilité, qui place la subjectivité en résonance avec les sensations qui émanent d'une société et d'une nature en constant bouleversement. Cette "grammaire des civilisations » est liée à ce moment précis des années 1796-1802. Helen-Maria Williams pouvait alors se rêver en égérie des temps nouveaux, avant que l'évolution du régime en lequel elle avait placé tant d'espoirs ne la rejette vers une marginalité mélancolique. Cette mélancolie est placée sous le signe du désenchantement, et produit une forme d'autocensure. Les œuvres majeures d'HelenMaria Williams sont publiées avant 1800, dans la décennie révolutionnaire. Sous l'Empire, elle ne prétend plus à aucun rôle public. La grammaire participe ensuite de la recomposition des savoirs ${ }^{31}$, devenant un domaine de spécialisation; tandis que les civilisations se figent dans une échelle hiérarchique et perdent de la fluidité qui était inhérente au projet originel. En œuvrant à sa dernière grande entreprise de traduction, celle des Voyages en Amérique méridionale de Alexandre de Humbold, traduction publiée à l'époque de la Première Restauration, en 1814, Helen-Maria Williams signe son appartenance à une autre époque. Commercialement, l'entreprise est d'ailleurs un échec retentissant. 


\section{NOTES}

1. Étienne DE FLACOURT, Histoire de la Grande Ile Madagascar, édition annotée, augmentée et présentée par Claude Allibert, Paris, Karthala, 2007, p. 86-87.

2. Relations des Jésuites, contenant ce qui s'est passé de plus remarquable dans les missions des Pères de la Compagnie de Jésus dans la Nouvelle-France : ensemble de volumes publiés à Paris chez Cramoisy entre 1641 et 1672 ; réédités en 1858 en trois volumes ; tome I (1611 - 1641), tome II (1642 - 1655), tome III (1656 - 1672).

On trouve des analyses récentes de cette collection dans La mission et le sauvage, huguenots et catholiques face aux populations indiennes, sous la direction de Nicole LEMAITRE, actes du $133^{\mathrm{e}}$ Congrès du CTHS, Québec, 2008 ; Paris, éditions du CTHS, 2009.

3. Jean-Luc CHAPPEY La Société des observateurs de l'Homme (1799-1804). Des anthropologues au temps de Bonaparte, Paris, Société des études robespierristes, 2002; id, "Entre communication et civilisation. Les langues et les dynamiques politiques entre République et Empire (1795-1808)», Colloque Le XIX ${ }^{e}$ siècle et ses langues, Société des études romantiques et des dix-neuviémistes. http ://etudes-romantiques.ish-lyon.cnrs.fr/wa_files/Langues-Chappey.pdf

4. Les volumes de La Décade... consultés pour cette communication sont ceux de la Bibliothèque de l'Arsenal, cotés 8 H 26375.

5. Cette présentation biographique doit beaucoup à l'ouvrage de Lionel D. WOODWARD, Une adhérente anglaise de la Révolution Française; Hélène-Marie Williams et ses amis, Paris, Honoré Champion, 1930. Plus récemment, nous avons la biographie de Deborah KENNEDY, Helen-Maria Williams and the Age of Revolution, Londres, Associated University Presses, 2002.

6. Albert MATHIEZ, La Révolution française et les étrangers. Cosmopolitisme et défense nationale, Paris, la Renaissance du livre, 1918.

7. Helen-Maria WiLliams, Nouveau voyage en Suisse, concernant une peinture de ce pays, de ses mours, et de ses gouvernements actuels, avec quelques traits de comparaison entre les usages de la Suisse et ceux de Paris moderne, Paris, Charles Pougens, nivôse an VI/janvier 1798. La carrière et l'influence d'Helen-Maria Williams sont à leur apogée sous le Directoire et au début du Consulat. Curieusement, les ouvrages qui lui sont consacrés accordent une part très réduite à cette période ; Woodward insiste sur les correspondants britanniques, tandis que Kennedy ne traite ces années cruciales qu'au prisme des relations avec Bonaparte. Voir Marcel DORIGNY et Bernard GAINOT, La Société des amis des noirs 1788-1799. Contribution à l'histoire de l'abolition de l'esclavage, Paris, Unesco, 1998, p. 351 et p. 368 ; et Bernard GAINOT, «La République et la Pastorale. Autour du Nouveau voyage en Suisse de Helen-Maria Williams (1798) », dans Gilles Bertrand et Pierre Serna (dir.), La République en voyage, 1770-1830, Rennes, PUR, 2013, p. 265-281.

8. Marron seconda Mirabeau dans la rédaction de ses pamphlets (notamment Appel aux bataves sur le stathoudérat.) et dans son entreprise de médiation entre le radicalisme anglais et la pensée révolutionnaire française.

9. Marc REGALDO, Un milieu intellectuel : la Décade philosophique (1794-1800), Paris, Honoré Champion, 1978 , t. 1, p. 267 et $s q$.

10. Il publie, en 1790, Discussions importantes débattues au parlement d'Angleterre par les plus célèbres orateurs depuis trente ans; puis, en 1796, Coup d'œil sur les courses de chevaux en Angleterre. Les frères Pictet fondèrent cette même année une entreprise spécialisée à Genève dans la traduction des ouvrages anglais : Bibliothèque britannique ou recueil extrait des ouvrages anglais périodiques ou autres. 
11. Sylvie KLEINMAN, Translation, the French language and the United Irishmen (1792-1804), PhD thesis, Dublin City University, 2005. http ://doras.dcu.ie/17994/

12. Sur cette entreprise éditoriale, voir Jean-Luc CHAPPEY, La Société des observateurs de l'homme, op. cit. et id., «La traduction comme pratique politique chez Antoine-Gilbert Griffet de Labaume (1756-1805) », dans Gilles Bertrand \& Pierre Serna (dir.), La République en voyage (1770-1830), op. cit., p. 233-250.

13. La Décade..., $\mathrm{n}^{\circ} 27 \mathrm{du} 30$ prairial an VII/18 juin 1799 ; pour l'analyse de cette entreprise éditoriale, voir Bernard GAINOT, « Lectures de Machiavel à l'époque du Directoire et du Triennio jacobin », dans Paolo Carta et Xavier Tabet (dir.), Macchiavelli nel XIXe XX secolo/ Machiavel aux XIX et $X X^{e}$ siècles, dipartimento di scienze giuridiche, Università di Trento, Milan, 2007, t. 67, p. 17-48.

14. Traduction de Paul et Virginie et de La chaumière indienne, parue à Londres en 1795 . Voir Anna BARKER, « Helen-Maria Williams's Paul and Virginia and the experience of mediated alterity », dans Luise Von Flotow (ed.), Translated women, University of Ottawa Press, 2011, p.?

15. Personnal narrative of travels to the equinoxial regions of the new continent, during the years 1799-1804, by Alexander de Humboldt and Aimé Bonpland, (en 7 volumes), Londres, 1818-1829.

16. Les extraits du Nouveau voyage en Suisse sont donnés le 10 frimaire an XI/ $1^{\mathrm{er}}$ décembre 1801, p. 424-427. Amaury Duval en avait par ailleurs commenté de larges extraits le 20 floréal an VI/9 mai 1798, p. 283-284, à partir de la traduction de Jean-Baptiste Say qui en est le traducteur.

17. La Décade..., $\mathrm{n}^{\circ} 11,20$ nivôse an IX/10 janvier 1801 ; rubrique "Livres nouveaux "; commentaire de Jean-Baptiste Say sur cet ouvrage dans le $n^{\circ} 13$ (10 pluviôse an IX/30 janvier 1801) ; puis un second commentaire dans le $n^{\circ} 14$ (20 pluviôse an IX/9 février 1801), à la rubrique « Littérature-Histoire ».

18. Ce patriote est Amodio Ricciardi, débarqué à Marseille à la fin du mois d'août 1799, arrivé à Paris le 21 décembre 1799. Il écrivit un mémoire qui circulait dans les milieux de réfugiés napolitains et qui est la source principale de l'essai d'Helen-Maria Williams. Pour l'analyse de ce mémoire, Anna Maria RAO, Esuli. L'emigrazione politica italiana in Francia (1792-1802), Naples, Guida, 1992, p. 441-447.

19. Vincenzo cuoco, Essai historique sur la Révolution de Naples, texte établi par Antonino de Francesco, édition bilingue, Les Belles Lettres, 2004.

20. Benjamin CONSTANT, Des réactions politiques, Paris, an V/1797.

21. La Décade..., Jean-Baptiste SAY, op. cit., p. 225.

22. Usurpation et chute du général Bonaparte par Sir Francis D'IVERNOIS. « Nec quies gentium sine armis, nec arma sine stipendiis, nec stipendia sine tributis », Tacite, Hist. lib. 4, Londres, décembre 1800 (frimaire an IX).

Analyse du texte et contexte dans Otto KARMIN, «Sir Francis d'Ivernois (1757 - 1842). Sa vie, son œuvre et son temps, précédé d'une notice sur son père François Henri d'Ivernois et sur la situation politique à Genève $(1748$ - 1768) », dans Revue historique de la Révolution française et de l'Empire, 1920.

23. Usurpation et chute..., ibid., p. 280. Cette citation est en fait une réplique de Benjamin Franklin à Lord Howe.

24. La Décade..., $\mathrm{n}^{\circ}$ du 20 brumaire an VIII/11 novembre 1799, p. 285-286.

25. Donatien-Alphonse-François DE SADE, Idée sur les romans, Évreux, Arlea, 1997, p. 28.

26. Compte rendu par Charles Theremin dans le numéro de La Décade... du 10 messidor an V/28 juin 1797 ; puis présentation d'un extrait du Précis sur l'établissement des colonies de Sierra-Leone et de Boulama, par Le Breton, dans le numéro du 30 ventôse an VI/20 mars 1798.

27. Bernard GAINOT, «La Décade et la colonisation nouvelle ", Annales historiques de la Révolution française, 339/2005, p. 99-116. 
28. La Décade..., $\mathrm{n}^{\circ}$ 22, 10 floréal an VII/29 avril 1799, rubrique «Biographie " (« Lettre de la citoyenne Helen-Maria Williams au citoyen J.B. Say, sur la mort du philanthrope Wadstrom ») : «La majeure partie de la vie de ce philanthrope fut consacrée à l'affranchissement et la civilisation de cette portion du genre humain qui habite le vaste continent de l'Afrique », p. 230.

29. La Décade..., $\mathrm{n}^{\circ} 15,30$ pluviôse an VII/18 février 1799, compte rendu de l'ouvrage suivant: Premiers principes de la langue anglaise par S. Baldwin. L'article est signé Masclet. Dans le $\mathrm{n}^{\circ} 36 \mathrm{du}$ 30 fructidor an VII/16 septembre 1799, le compte rendu de l'ouvrage de Sylvestre de Sacy, Principes de grammaire générale, mis à la portée des enfants, et propres à servir d'instruction à l'étude de toutes les langues, s'inscrit dans le prolongement de ces présupposés théoriques : il faut établir une nomenclature de base pour les sciences du langage et des idées.

30. Ibid.

31. Jean-Luc CHAPPEY, "The 'New Elites' ", dans David Andress (ed.), Oxford Handbook of the French Revolution, Oxford University Press, 2013, p. ?

\section{RÉSUMÉS}

La question de la traduction est essentielle dans le journal des Idéologues, La Décade philosophique, littéraire et politique. C'est tout à la fois une extrême attention portée aux modalités du langage, et le souci délibéré de faire de celui-ci le marqueur d'un processus de civilisation. J'ai retenu une personnalité de premier plan au sein de l'équipe éditoriale de ce journal, Helen-Maria Williams, qui est tout à la fois traductrice, et auteur traduit. C'est une médiatrice essentielle au temps de la République Directoriale.

Elle est alors particulièrement liée à Jean-Baptiste Say, qui consacre des comptes rendus approfondis et élogieux de deux de ses ouvrages majeurs : le Nouveau voyage en Suisse (1798) et Aperçu de l'état, des mœurs et des opinions dans la République française, vers la fin du XVIII ${ }^{e}$ siècle, (1801). L'accent est mis sur les valeurs de référence de l'auteure, qui sont en opposition complète avec le nationalisme contre-révolutionnaire anglais, dont Edmund Burke est le principal représentant. Parallèlement, la propre contribution de Helen-Maria Williams à la promotion d'une nouvelle sensibilité littéraire se remarque surtout à travers ses propres comptes rendus de romans anglais, inspirés par Laurence Sterne et le marquis de Sade.

Plus largement, ces contributions participent d'une œuvre de médiation entre des cultures dont il s'agit de dépasser les antagonismes, pour en percevoir les signes universels, produit d'une réflexion sur la langue, et sur la pratique narrative d'une nouvelle sensibilité, qui place la subjectivité en résonance avec les sensations qui émanent d'une société et d'une nature en constant bouleversement. C'est une entreprise de « grammaire des civilisations ».

The issue of translation is essential in the Idéologues newspaper, La Décade philosophique, littéraire et politique. It altogether takes an extreme care of language methods and demonstrates the deliberate willingness to use language as a milestone in a civilisation process. I have chosen a leading figure within the editorial team of this newspaper, Helen-Maria Williams, who is at the same time both a translator but also a translated author. She is an essential mediator during the République Directoriale period.

At the time, she is especially closed to Jean-Baptise Say, who drafted in-depth and laudatory reports on two of her major publications : le Nouveau voyage en Suisse (1798) and Aperçu de l'état, des mœurs et des opinions dans la République française, vers la fin du XVIII siècle, (1801). Say 
emphasizes Williams' reference values, which are in total contradiction with English counterrevolutionary nationalism, embodied by Edmund Burke as the main representative. In parallel, Helen-Maria's own contribution to the promotion of a new literary trend transpires particularly through her own reports on English novels, inspired by Laurence Sterne and Ideas on novels (whose author is the Marquis of Sade).

More generally, these contributions lead to a mediation work between cultures, which antagonisms are overcome in order to perceive their universality, as the result of a thinking process on language as well as on narrative practice entailing a new trend, where the individual echoes both society's lifestyle and an ever-evolving nature. It is an innovative undertaking leading to a "grammaire des civilisations" (universal system of values for dialogue between cultures).

INDEX

Keywords : La Décade philosophique et politique, the philosophical and political decade, civilisation, cultural mediation, English novels, new colonisation, Directoire, translation art Mots-clés : La Décade philosophique et politique, civilisation, médiation culturelle, romans anglais, nouvelle colonisation, Directoire, art de la traduction

\section{AUTEUR \\ BERNARD GAINOT}

IHMC - Institut d'histoire moderne et contemporaine

Université Paris 1 Panthéon-Sorbonne 